Original article

\title{
ANTIOXIDANT EFFECTS OF SILYMARIN ON ISCHAEMIA- REPERFUSION INJURIES OF THE RABBIT RETINA
}

\author{
A. ALIABADI ${ }^{1}$, A. JAVAHERI ${ }^{1}$, A. ESFANDIARI ${ }^{2} \&$ H. GHAHRAMANI ${ }^{1}$ \\ ${ }^{1}$ Department of Clinical Studies; ${ }^{2}$ Department of Basic Sciences, \\ School of Veterinary Medicine, Kazerun Branch, Islamic \\ Azad University, Kazerun, Iran
}

\section{Summary}

Aliabadi, A., A. Javaheri, A. Esfandiari \& H. Ghahramani, 2016. Antioxidant effects of silymarin on ischaemia-reperfusion injuries of the rabbit retina. Bulg. J. Vet. Med., 2016, 19, No 4, 290-298.

\begin{abstract}
Retinal ischaemia is known as the main reason of blindness in disease such as closed angle glaucoma and diabetes. The aim of this study was to investigate the protective effect of silymarin in injuries induced by ischaemic-reperfusion of rabbit eyes. Thirty male adult New Zealand white rabbits were divided into three groups of ten animals each. All animals in the two experimental groups underwent bilateral common carotid ligation for $60 \mathrm{~min}$. After 48 hours reperfusion all animals were sacrificed. Silymarin-treated group received $250 \mathrm{mg} / \mathrm{kg}$ of silymarin $48 \mathrm{~h}$ before surgery, placebo-treated group received same volume of normal saline as experimental group I, without any medication and control group was non-ischaemic and untreated. After processing, retinal histology samples were investigated by electron microscopy. The concentration of lipid peroxides in retinas was measured. Histology results of this experiment revealed that in the reperfusion groups, the group that received no treatment had major signs and silymarin group had minor signs of pathology. The loss of outer segments, scattered and disorganised inner segments were observed in placebo-treated group, but in silymarintreated group the outer segment was normal and inner segments were organised. On the other hand, some pyknotic, condensed, and karyorrhexis nuclei in the outer nuclear layer were obvious in placebo-treated group. Measurement of concentration of lipid peroxides in the retina also showed a significant decrease in malondialdehyde (MDA) level in silymarin-treated group.
\end{abstract}

Key words: ischaemia-reperfusion, rabbits, silymarin

\section{INTRODUCTION}

Eyes are the organs of vision. They detect light and convert it into electro-chemical impulses in neurons. In higher organisms the eye is a complex optical system that collects light from the surrounding environment, regulates its intensity through a diaphragm, focuses it through an adjustable assembly of lenses to form an image, converts this image into a set of electrical signals, and transmits these signals to the brain through complex neural pathways that connect the eye via the optic nerve to 
the visual cortex and other areas of the brain (Land \& Fernald, 1992). On the other hand, the retina is a multi-layered sensory tissue that lines the back of the eye. It contains millions of photoreceptors that capture light rays and convert them into electrical impulses. These impulses travel along the optic nerve to the brain where they are turned into images.

Ischaemia-reperfusion ( $\mathrm{I} / \mathrm{R})$ is a wellrecognised pathological condition that is characterised by an initial deprivation of blood supply to an area or organ followed by subsequent vascular restoration and concomitant reoxygenation of downstream tissue (Eltzschig \& Eckle, 2011). I/R can develop as a consequence of trauma, hypertension, shock, sepsis, organ transplantation, or bypass surgery leading to endorgan failure such as acute renal tubular necrosis, bowel infarction, and liver failure. I/R can also occur under various complications of vascular diseases such as stroke and myocardial infarction (Duehrkop \& Rieben, 2014).

Retinal ischaemia is a common cause of visual impairment and blindness. Ischaemia is a term used to describe a tissue whose blood supply has been reduced to an insufficient level. Lack of oxygen in the retinal tissue may lead to retinal cell death or cell dysfunction, and thereby result in reduced vision. At the cellular level, ischaemic retinal injury consists of a self-reinforcing destructive cascade involving neuronal depolarisation, calcium influx and oxidative stress initiated by energy failure and increased glutamatergic stimulation (Minhas \& Anand, 2011). Retinal ischaemia-reperfusion models are used to study several components of various eye disease pathologies such as retinal vein occlusion, diabetic retinopathy, and glaucoma (Hirooka et al., 2006; Zheng et al., 2007). The IR model has been widely used for studying retinal neuronal cell damage after ischaemic insult and consists of transient ischaemia followed by natural reperfusion leading to an inflammatory and neurodegenerative response in the intact retina (Osborne et al., 2004). Histologic analysis demonstrated that the IR injury causes selective neuronal loss indicated by reduced thickness of retinal layers including the ganglion cell layer, inner nuclear layer, and inner plexiform layer (Husain et al., 2009). A recent study demonstrated that IR also induced vascular abnormalities such as capillary dropout after 8 to 14 days of reperfusion and concluded that these vascular changes occurred after neuronal loss (Zheng et al., 2007). Retinal I/R interrupts blood flow to retinas and results in a deficiency of oxygen and other nutrients, whereas the subsequent reperfusion exacerbates the tissue damage because of the generation of reactive oxygen species (ROS) that lead to oxidative stress and inflammation (Hirooka et al., 2006; Antonetti et al., 2012). Oxidative stress plays a vital role in retinal neuronal injury (Zheng et al., 2007) therefore; the identification of a potential antioxidant therapy for $\mathrm{I} / \mathrm{R}$-induced damage has attracted intense interest.

Medicinal plants are considered rich resources of ingredients that can be used in drug development and synthesis. Moreover, some plants are considered as important sources of nutrition and, as a result, are recommended for their therapeutic values (Hassan, 2012). Silymarin (SM) is a phytochemical isolated from the milk thistle plant Silybum marianum. Silymarin consists of a mixture of three bioflavonoids found in the fruit, seeds and leaves of the plant: silybin, silydianin and silychristin. Silymarin is used for the treatment of numerous liver disorders charac- 
terised by degenerative necrosis and functional impairment. The compound also protects hepatocytes from injury caused by ischaemia, radiation, iron overload and viral hepatitis and the antioxidant effect of silymarin in the renal ischaemiareperfusion injury and renal toxicity has been investigated successfully before (Vogel \& Trost, 1975). It should be noted that SM can contribute to the antioxidant defences in different ways: first, by direct free radical scavenging and second, by preventing free radical formation by inhibiting specific enzymes responsible for free radical production, or by maintaining the integrity of electron-transport chain of mitochondria in stress conditions. A third mechanism is the participation in the maintenance of optimal redox status of the cell by activating a range of antioxidant enzymes and non-enzymatic antioxidants, mainly via transcription factors, including $\mathrm{Nrf} 2$ and NF- $\kappa \mathrm{B}$. Finally, it activates an array of vitagenes, responsible for the synthesis of protective molecules, including HSP, thioredoxin, sirtuins etc., and providing additional protection in stress conditions. In most studies pure silybin, as the main component of SM, was used, however, in some cases SM also showed antioxidant action in vivo.

The aim of this study was to survey the protective antioxidant effect of silymarin in injuries induced by ischaemia-reperfusion of rabbits' eyes.

\section{MATERIALS AND METHODS}

\section{Experimental design}

Thirty male adult New Zealand white rabbits, weighing $1-1.5 \mathrm{~kg}$ were used in this study. The rabbits were divided into three equal groups: silymarin-treated group (I) which received $250 \mathrm{mg} / \mathrm{kg}$ silymarin daily from two days before surgery until opera- tion, placebo-treated group (II) that received the same volume of normal saline as experimental group I, without any medication and control group (nonischaemic, untreated). The animals were allowed free access to standard food diet and water in the animal laboratory. The rabbits were quarantined for 10 days at $22-24{ }^{\circ} \mathrm{C}$ with a $12 \mathrm{~h}$ light $/ 12 \mathrm{~h}$ dark cycle before beginning the experimental process for adaptation. The animals in surgery groups were anaesthetised with intraperitoneal injection of $13 \mathrm{mg} / \mathrm{kg}$ xylazine hydrochloride and $70 \mathrm{mg} / \mathrm{kg}$ ketamine hydrochloride (Alfasan, Woerden, Holand). Hair was clipped in the lower area of theneck in group 1 and 2. The depilated skin was prepared for aseptic surgery. A $2 \mathrm{~cm}$ incision was created in the ventral area of the neck and common carotid arteries on both sides were exposed gently and clamped in order to induce ischaemia in both retinas. After $60 \mathrm{~min}$, the clamps were removed for reperfusion of the retina. Then the skin was sutured routinely and rabbits were transferred back to the animal laboratory. All animals from the silymarin-treated group received orally $250 \mathrm{mg} / \mathrm{kg}$ silymarin powder (SigmaAldrich, Germany) 48 h before surgery. Rabbits from the placebo-treated group received normal saline orally as placebo in the same period of time. At the end of the treatment, all rabbits were euthanised with intraperitoneal injection of sodium thiopental overdose (Abbott Laboratories, USA).

Both eyes of all rabbits were removed and then placed in 4\% glutaraldehyde fixative for $4 \mathrm{~h}$. The retina was separated near the optic disc and processed for transmission electron microscope (Zarenezhad et al., 2013). The procedure was approved by the Ethical committee of Islamic Azad University, Kazeroon branch. 


\section{Electron microscopic examination}

For electron microscopic examination, the retina samples were fixed in $2.5 \%$ glutaraldehyde buffered with $0.2 \mathrm{M} \mathrm{NaH}_{2} \mathrm{PO}_{4}+$ $\mathrm{NaHPO}_{4}(\mathrm{pH}=7.4)$ and postfixed in $1 \%$ $\mathrm{OsO}_{4}$. After dehydration in acetone, they were embedded in Araldite CY 212. U1trathin sections were stained with uranyl acetate and lead citrate and examined by a Zeiss Libra 120 transmission electron microscope (Emre et al., 2009).

\section{Analysis of lipid peroxides}

After enucleation of eyes, ten retinas from each group were removed for malondialdehyde (MDA) assay. The lipid peroxide concentration was determined by the method described by Ohkawa et al. (1979) which measures thiobarbituric acid reactivity by the amount of MDA formed during acid hydrolysis of the lipid peroxide compound.

\section{Statistical analysis}

Lipid peroxide concentration was analyzed by one-way analysis of variance (ANOVA) and $\mathrm{P}<0.05$ was considered statistically significant.

\section{RESULTS}

Evaluation of pictures obtained from electron microscopy in this research showed that photoreceptor layer in control group consisted of outer segment, inner segment, mitochondria, outer limiting membrane and outer nuclear layer. The outer segment of the control group was comprised of bimembranous discs. The inner segment in control group contained round, oval and cylindrical mitochondria that were presented near the outer segment and also, the inner segment appeared normal without vacuole. In this group, the outer limiting membrane seemed normal and included zonula adherens junction; the outer nuclear layer consisted of round to oval nuclei with normal scattered heterochromatin in nuclei (Fig. 1).

The placebo-treated group had major signs of pathology. In this group the loss of outer segments, scattered and disorganized inner segments was observed. In addition, the large and small vacuoles were observed in the inner segment (Fig. 2). However, the inner segment region looked abnormal and diffused. Some pyknotic, condensed and karyorrhexis nuclei exist in the outer nuclear layer (Fig. 3). The outer limiting membrane appeared normal in this group.

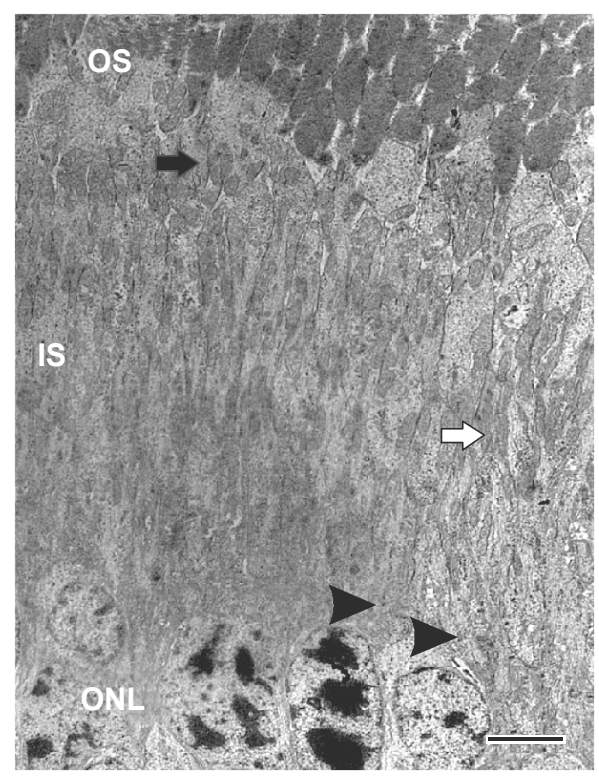

Fig. 1. Electromicrograph of the photoreceptor layer in control group: outer segment (OS), inner segment (IS), mitochondrial layer (white arrows), outer limiting membrane (arrowheads); outer nuclear layer $(\mathrm{ONL})$. Bar $=1800 \mathrm{~nm}$. 


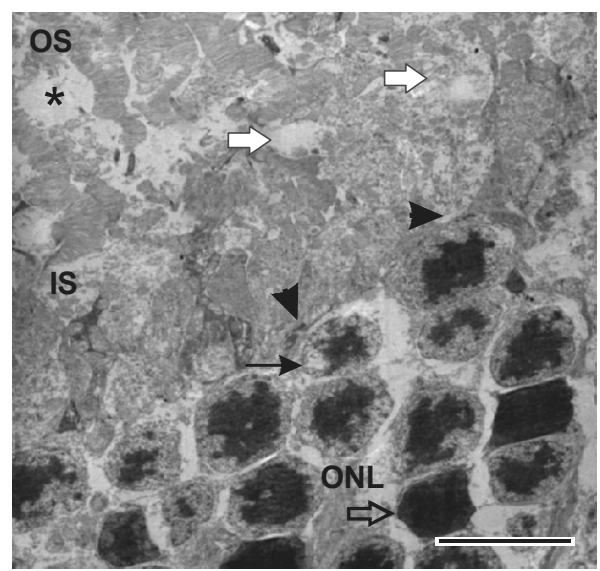

Fig. 2. Electromicrograph of the photoreceptor layer in the reperfusion group: outer segment (OS), outer segment loss (asterisk), the disorganised inner segment (IS), the mitochondria have disappeared, the vacuole (white arrows), outer limiting membrane (arrowheads), outer nuclear layer (ONL), condensed (hollow arrow) and pyknotic (thin arrow) nuclei. Bar $=1800 \mathrm{~nm}$.

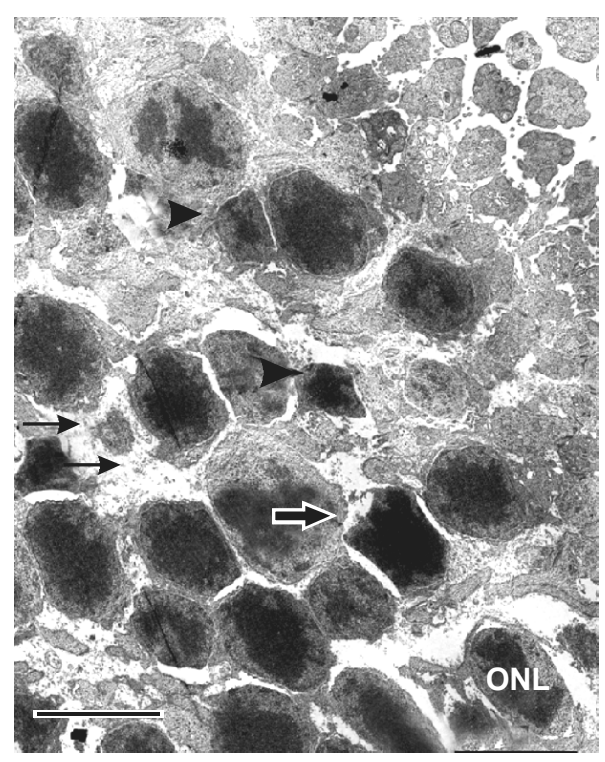

Fig. 3. Electromicrograph of the outer nuclear layer in reperfusion group: outer nuclear layer (ONL), condensed nuclei (thick arrow), pyknotic nuclei (arrowheads), karyorrhexis nuclei (thin arrows). Bar $=1500 \mathrm{~nm}$.
The minor signs of histopathology were seen in silymarin-treated group. The outer segment appeared normal; organised inner segments were seen (Fig. 4).

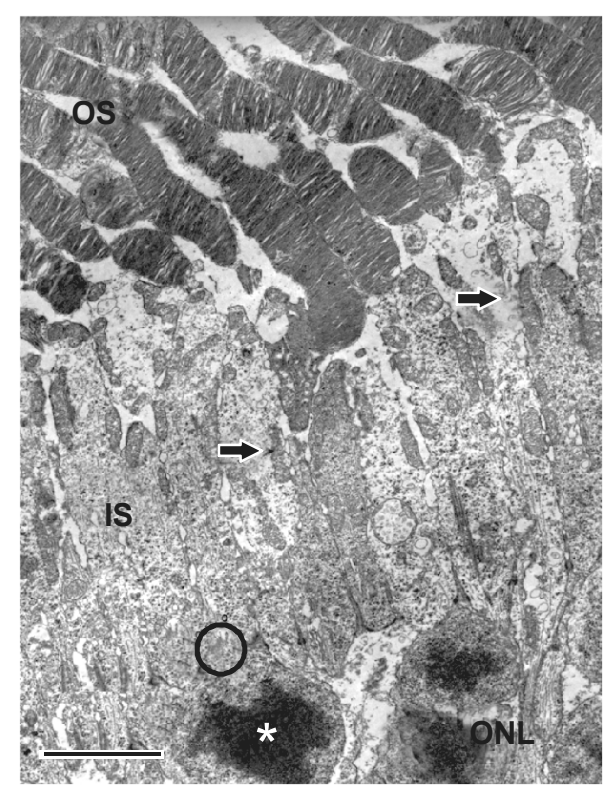

Fig. 4. Electromicrograph of the photoreceptor layer in silymarin-reperfusion group - normal outer segment (OS), organised inner segment (IS), mitochondria (arrows), outer limiting membrane (circle), outer nuclear layer (ONL), condensed nuclei (asterisk). Bar $=1800 \mathrm{~nm}$.

The outer limiting membrane appeared normal but condensed nuclei in the outer nuclear layer were observed (Fig. 5).

In control group, the lipid peroxide TBA value was $138 \pm 10.8 \mathrm{nmol} / \mathrm{g}$ wet weight. Lipid peroxide concentration increased significantly to $278 \pm 15.6 \mathrm{nmol} / \mathrm{g}$ in placebo-treated group, $48 \mathrm{~h}$ after reperfusion compared to normal group while this amount dropped significantly to $179 \pm 12.3 \mathrm{nmol} / \mathrm{g}$ in silymarin-treated group that received oral silymarin two days before reperfusion compared to placebo-treated group (Fig. 6). 


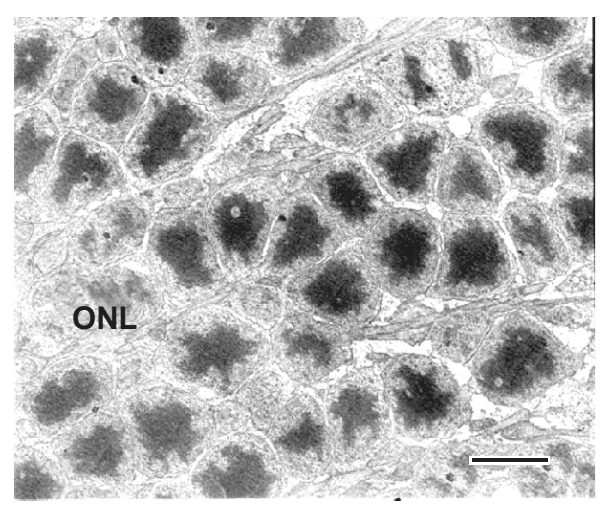

Fig. 5. Electromicrograph of the outer nuclear layer in silymarin-reperfusion group. Outer nuclear layer with condensed nuclei (ONL). $\mathrm{Bar}=1500 \mathrm{~nm}$.

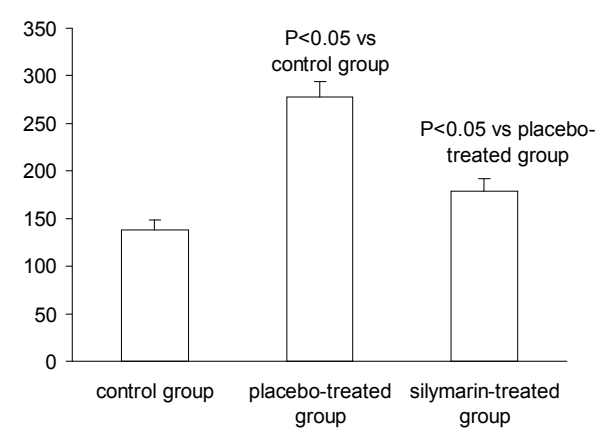

Fig. 6. Retinal malondialdehyde concentrations (nmol/g retina) in the three groups of rabbits 48 hours after the 60-min ischaemiareperfusion injury (mean $\pm \mathrm{SEM}, \mathrm{n}=10$ ).

\section{DISCUSSION}

Ischaemia-reperfusion is a pathological condition that is characterised by an initial deprivation of blood supply to an area or organ followed by the subsequent restoration of perfusion and concomitant reoxygenation (Osborn et al., 2004). However, surprisingly little is known about effects of ischaemia-reperfusion on microstruc- ture events in retina. The present study was undertaken to test the hypothesis that silymarin, as an antioxidant, would protect the retina from ischaemia reperfusion injuries. In this study, we investigated the antioxidant effect of silymarin in injuries induced by ischaemia-reperfusion of rabbits eyes. Disorganisation in retinal structure in placebo-treated group was evident in comparison with silymarin-treated group. So the loss of outer segments, scattered and disorganised inner segments was observed in placebo group, but in silymarin group the outer segment was normal and inner segments were organised.

Ischaemia-reperfusion is one of the events producing significant amounts of free radicals. Free radicals and oxidants play a dual role as both toxic and beneficial compounds, since they can be either harmful or helpful to the body. When an overload of free radicals cannot gradually be destroyed, their accumulation in the body generates a phenomenon called oxidative stress. In the retina, long-term exposure to oxidative stress can inhibit mitosis in the retinal pigment epithelium and choroids, damage the photoreceptor outer segments, and has been associated with lipid peroxidation (Myatt, 2006). The retina is particularly susceptible to oxidative stress because of its high consumption of oxygen, its high proportion of polyunsaturated fatty acids, and its exposure to visible light. In vitro studies have consistently shown that photochemical retinal injury is attributable to oxidative stress and that the antioxidant vitamins A, C, and $\mathrm{E}$ protect against this type of injury (Beatty et al., 2000). In our study the high rate of lipid peroxides in placebo group implies that the high concentration of free radicals is present in reperfused retina. Antioxidant action of SM has been shown in various model systems. According to 
our information, so far there has been no research carried out to survey the effect of silymarin in ischaemia-reperfusion in eye. The effect of other medicines in injuries induced by ischaemia-reperfusion in eyes and the effect of silymarinin injuries induced by ischaemia-reperfusion in other parts of the body have been examined previously by other researchers. For instance, Rao \& Viswantha (2007) by surveying the cardioprotective activity of silymarin in ischaemia/reperfusion-induced myocardial infarction in albino rats reported that silymarin protected the endogenous antioxidant enzymes, suppressed the neutrophil infiltration during ischaemia-reperfusion. Turgut et al. (2008) investigated the antioxidant and protective effects of silymarin on ischaemia and reperfusion injury and reported that animals that were subjected to ischaemiareperfusion exhibited significant increase in serum urea, creatinine, and cystatin $\mathrm{C}$ levels compared with the rats treated with silymarin prior to the ischaemia-reperfusion process. Emre et al. (2009) compared the protective effects of prostaglandin analogues in the ischaemia and reperfusion model of rabbits eyes and reported that prostaglandin analogues-treated groups had fewer apoptotic cells in all retinal layers than the saline-treated ischaemic group. It has also been reported that prostaglandin analogues decreased vitreous xanthine oxidase activity significantly compared to the saline-treated group.

Hou et al. (2010) studied the preventive effect of silymarin in cerebral ischaemia-reperfusion-induced brain injury in ratsand reported that silymarin protected rats against $\mathrm{CI} / \mathrm{R}$-induced stroke injury by amelioration of the oxidative and nitrosative stresses and inflammationmediated tissue injury through impeding the activation of proinflammatory tran- scription factors in the upregulation of proinflammatory proteins and cytokines in stroke-damaged sites. By surveying the effect of silymarin on mesenteric ischaemia-reperfusion injury, Demir et al. (2014) reported that silymarin therapy attenuates the oxidative and intestinal damage induced by ischaemia-reperfusion injuries. The data obtained in the present study showed that silymarin protected the different retinal parts which may relate to its antioxidant effects in prevention of oxidative stress. The outer segment loss was obvious in placebo group. Probably, oxidative stress of ischaemia-reperfusion in the outer segment is associated with double bond breaking of 11-cis-retinal during cis-trans isomerisation. This photochemical reaction has been reported to cause the formation of oxygen free radicals. Oxygen free radicals can also form protein peroxides (Williams, 2008). The changes of the primary structure of protein may induce loss of outer segment layer. The scattered and disorganised inner segment occurred in placebo group. Therefore, these changes give rise to mitochondrial damage and reduce oxidative metabolism which may also lead to photo oxidative damage by reactive oxygen species formation. The pyknotic nuclei were observed in the placebo group. These changes are responses to injury or damage cells. All these histological changes decreased in silymarin-treated compared to the placebo group. On the other hand, the decrease of free radicals in silymarin group compared to placebo group may reveal the positive effect of silymarin on the reduction of free radicals in retina after 48 hours.

In conclusion, the present investigation showed that silymarin treatment was useful in the functional recovery of the retina after injury by the ischaemia reper- 
fusion, and also, this finding may provide a basis for the development of novel therapeutic strategies for protection against the damages caused by ischaemia-reperfusion.

\section{REFERENCES}

Antonetti, D. A., R. Klein \& T. Gardner, 2012. Diabetic retinopathy. New England Journal of Medicine, 366, 1227-1239.

Beatty, S., H. Koh, D. Henson \& M. Boulton, 2000. The role of oxidative stress in the pathogenesis of age-related macular degeneration. Survey of Ophthalmology, 2, 115-134.

Demir, M., R. Amanvermez, A. Polat \& I. Karabicak, 2014. The effect of silymarin on mesenteric ischemia-reperfusion injury. Medical Principles and Practice, 23, 140-144.

Duehrkop, C. \& R. Rieben, 2014. Ischemia/reperfusion injury: Effect of simultaneous inhibition of plasma cascade systems versus specific complement inhibition. biochemical Pharmacology, 88, 12-22.

Eltzschig, H. K. \& T. Eckle, 2011. Ischemia and reperfusion - from mechanism to translation. Nature Medicine, 17, 1391-1401.

Emre, S., M. Gul, B. Ates, B. Koc \& A. Erdogan, 2009. Comparison of the protective effects of Prostaglandin analogues in the ischemia and reperfusion model of rabbit eyes. Experimental Animals, 5, 505-513.

Hassan, B., 2012. Medicinal plants (importance and uses). Pharmaceutica Analytica Acta, 10, 2153-2435.

Hirooka, K., O. Miyamoto, P. Jinming, Y. Du, T. Itano, T. Baba, M. Tokuda \& F. Shiraga, 2006. Neuroprotective effects of Dallose against retinal ischemia-reperfusion injury. Investigative Ophthalmology and Visual Science, 47, 1653-1657.

Hou, Y. C., K. T. Liou, C. M. Chern, Y. H. Wang, J. F. Liao, S. Chang, Y. H. Chou \& Y. C. Shen, 2010. Preventive effect of silymarin in cerebral ischemia-reperfusioninduced brain injury in rats possibly through impairing NF- $\kappa$ B and STAT-1 activation. Phytomedicine, 12, 963-973.

Husain S., D. Potter \& C. Crosson, 2009. Opioid receptor-activation: Retina protected from ischemic injury. Investigative Ophthalmology and Visual Science, 50, 3853-3859.

Land, M. \& R. Fernald, 1992. The evolution of eyes. Annual Review of Neuroscience, 15, 1-29.

Minhas, G. \& A. Anand, 2011. Animal models of retinal ischemia. Neurochemistry International, 53, 154-169.

Myatt, L., 2006. Placental adaptive responses and fetal programming. The Journal of Physiology, 572, 25-30.

Ohkawa, H., N. Ohishi \& K. Yagi, 1979. Assay for lipid peroxidation in animal tissues by thiobarbituric acid reaction. Annals of Biochemistry, 95, 351-358.

Osborne, N., R. Casson, J. Wood, G. Chidlow, M. Graham \& J. Melena, 2004. Retinal ischemia: Mechanisms of damage and potential therapeutic strategies. Progress in Retinal and Eye Research, 1, 91-147.

Rao, P. \& R. Viswantha, 2007. Cardioprotective activity of silymarinin ischemia-reperfusion-induced myocardial infarctionin albino rats. Experimental \& Clinical Cardiology, 4, 179-187.

Turgut, F., O. Bayrak, F. Catal, R. Bayrak, A. F. Atmaca, A. Koc, A. Akbas, A. Akcay \& D. Unal, 2008. Antioxidant and protective effects of silymarin on ischemia and reperfusion injury in the kidney tissues of rats. International Urology and Nephrology, 2, 453-460.

Vogel, G. \& W. Trost, 1975. Anti-phalloidinaktivität der silymarine silybin und disilybin. Arzneimittelforschung, 25, 392-393.

Williams, D. L., 2008. Oxidative stress and the eye. Veterinary Clinics of North America: Small Animal Practice, 38, 179-192.

Zarenezhad, A., A. Esfandiari \& A. Aliabadi, 2013. Preventive effects of silymarin in retinal intoxication with methanol in rat: Transmission electron microscope study. 
Antioxidant effects of silymarin on ischaemia-reperfusion injuries of the rabbit retina

African Journal of Pharmacy and Pharmacology, 25, 1757-1761.

Zheng, L., B. Gong, D. A. Hatala \& T. S. Kern, 2007. Retinal ischemia and reperfusion causes capillary degeneration: similarities to diabetes. Investigative Ophthalmology and Visual Science, 48, 361-367.

Paper received 29.05.2015; accepted for publication 15.06.2015

\section{Correspondence:}

Ali Aliabadi

Department of Clinical Studies, School of Veterinary Medicine, Kazerun Branch,

Islamic Azad University,

Kazerun, Iran

e-mail: aaliabadi@gmail.com 